\title{
GLOBAL TRANSVERSAL STABILITY OF EUCLIDEAN PLANES UNDER SKEW MEAN CURVATURE FLOW EVOLUTIONS
}

\author{
ZE LI
}

\begin{abstract}
In this paper, we prove that 2 dimensional transversal small perturbations of d-dimensional Euclidean planes under the skew mean curvature flow lead to global solutions which converge to the unperturbed planes in suitable norms. And we clarify the long time behaviors of the solutions in Sobolev spaces.
\end{abstract}

\section{InTRODUCTION}

Let $\Sigma$ be a $d$-dimensional oriented manifold and $(\mathcal{N}, h)$ be a $(d+2)$-dimensional oriented Riemannian manifold. Assume that $\mathbb{I}$ is an interval containing $t=0$, and $F: \mathbb{I} \times \Sigma \rightarrow \mathcal{N}$ is a family of immersions. For each given $t \in \mathbb{I}$, denote $\Sigma_{t}=F(t, \Sigma)$ the submanifold and $\mathbf{H}(F)$ its mean curvature vector. Denote the tangent bundle of the submanifold by $T \Sigma_{t}$ and the normal bundle by $N \Sigma_{t}$ respectively. There exists a natural induced complex structure $J(F)$ for $N \Sigma_{t}$ (rank two) via simply rotating a vector in the normal space by $\frac{\pi}{2}$ positively. To be precise, for any point $z=F(t, x) \in \Sigma_{t}$ and normal vector $\nu \in N_{z} \Sigma_{t}$, define $J(F)$ by letting $J(F) \nu \perp \nu=0$ and $\omega\left(F_{*}\left(e_{1}\right), \ldots, F_{*}\left(e_{d}\right), \nu, J(F) \nu\right)>0$, where $\omega$ is the volume form of $\mathcal{N}$ and $\left\{e_{1}, \ldots, e_{d}\right\}$ is an oriented basis of $T \Sigma_{t}$. We shall call the binormal vector $J(F) \mathbf{H}(F) \in N \Sigma_{t}$ the skew mean curvature vector. And the skew mean curvature flow (SMCF) for immersions $F: \mathbb{I} \times \Sigma \rightarrow \mathcal{N}$ is defined by

$$
\left\{\begin{array}{l}
\partial_{t} F=J(F) \mathbf{H}(F) \\
F(0, x)=F_{0}(x), x \in \Sigma
\end{array}\right.
$$

In view of geometric flows, the SMCF evolves a codimension 2 submanifold along its binormal direction with a speed given by its mean curvature. It is remarkable that SMCF was historically derived from both physics and mathematics. The motivations from physics are vortex filament equations (VFE), the localized induction approximation (LIA) of the hydrodynamical Euler equations, and asymptotic dynamics of vortices in superfluidity and superconductivity.

In fact, the 1-dimensional SMCF in the Euclidean space $\mathbb{R}^{3}$ is the vortex filament equation

$$
\partial_{t} u=\partial_{s} u \times u, u:(s, t) \in \mathbb{R} \times \mathbb{R} \longmapsto u(s, t) \in \mathbb{R}^{3},
$$

Key words and phrases. skew mean curvature flow, motion of membranes, global stability . 
where $t$ denotes time, $s$ denotes the arc-length parameter of the curve $u(t, \cdot)$, and $\times$ denotes the cross product in $\mathbb{R}^{3}$. The VFE describes the free motion of a vortex filament, see Da Rios [4] and Hasimoto [9].

The SMCF also appears in the study of asymptotic dynamics of vortices in the context of superfluidity and superconductivity. For the Gross-Pitaevskii equation, which models Bose-Einstein condensates, physicists conjecture that the vortices would evolve along the SMCF. This was first verified by Lin [18] for the vortex filaments in $\mathbb{R}^{3}$. Similar phenomena were also observed for other PDEs, for example, it was shown for the Ginzburg-Landau heat flow that the energy asymptotically concentrates on the codimension 2 vortices whose motion is governed by the mean curvature flow.

The other motivation is the LIA of hydrodynamical Euler equations, which describes the limit of a generalized Biot-Savart formula. In fact, let $M \subset \mathbb{R}^{d}$ with $d \geq 3$ be a closed oriented submanifold of codimension 2. Consider the vorticity 2-form $\eta_{M}$ supported on this submanifold: $\eta_{M}=C \cdot \delta_{M}$. We shall call $M$ a higher dimensional vortex filament or membrane. Then for any dimension $d \geq 3$ the divergence-free vector field $v$ in $\mathbb{R}^{d}$ satisfying curlv $=\eta_{M}$ is given by a generalized Biot-Savart formula, which only holds for points away from $M$. In order to derive the formula of $v$ for points within $M$, one defines truncation vector field $v_{\epsilon}$ by the above generalized Biot-Savart formula and some truncation. It was shown that the limit of $(\ln \epsilon)^{-1} v_{\epsilon}(x)$ for $x \in M$ as $\epsilon \rightarrow 0$ is the skew mean curvature vector of $M \hookrightarrow \mathbb{R}^{d}$. Hence, the LIA approximation for a vortex membrane (or higher filament) $M$ in $\mathbb{R}^{d}$ up to a suitable scaling coincides with the SMCF. These facts were discovered by Shashikanth [25] and generalized by Khesin [15].

SMCF also emerges in different mathematical problems. In study of nonlinear Grassmannians, Haller-Vizman [7] noted that SMCF is the Hamiltonian flow of the volume functional on the space consisting of all co-dimensional 2 immersions of a given Riemannian manifold. Notice that this space admits a generalized Marsden-Weinstein symplectic structure [24] which provides the volume form. The SMCF of surfaces in $\mathbb{R}^{4}$ is included by a vast energy conserved motion project raised by Lin and his collaborators [19]. Terng [30] also proposed SMCF under the name of star MCF.

Let us describe the non-exhaustive list of works on SMCF. The case $d=1$, i.e. the VFE, has been intensively studied by many authors from many views. We recommend the reader to read the survey Vega [32] and Gomez's thesis [6] for VFE. The works on the case $d \geq 2$ are much less. Song-Sun [27] proved local existence of SMCF for $F: \Sigma \rightarrow \mathbb{R}^{4}$ with compact oriented surface $\Sigma$. This was generalized by Song [28] to $F: \Sigma \rightarrow \mathbb{R}^{d+2}$ with compact oriented surface $\Sigma$ for all $d \geq 2$. Khesin-Yang [16] constructed an example showing that the SMCF can blow up in finite time. Jerrard proposed a notion of weak solutions to the SMCF in [10]. Song [26] proved that the Gauss map of a $d$ dimensional $\mathrm{SMCF}$ in $\mathbb{R}^{d+2}$ satisfies a Schrödinger map flow equation (see e.g. $[2,3,5,31]$ ). 
For $d \geq 2$, the global existence theory of SMCF is largely open even for small data. This is what we aim to solve in this paper. Let us consider the case $\Sigma=\mathbb{R}^{d}, \mathcal{N}=\mathbb{R}^{d+2}$ in SMCF. It is easy to see $F\left(t, x_{1}, \ldots, x_{d}\right)=\left(x_{1}, \ldots, x_{d}, 0,0\right)$ is a solution of SMCF, i.e. the d-dimensional planes. We consider the transversal perturbations of this plane, i.e., we seek for a graph like solution of the form

$$
F(t, x)=\left(x_{1}, \ldots, x_{d}, u_{1}(t, x), u_{2}(t, x)\right) .
$$

Our following main theorem states that if the initial perturbation $u_{1}(0, \cdot), u_{2}(0, \cdot)$ is sufficiently small, then SMCF has a global graph like solution and the asymptotic behaviors can be clearly determined.

In fact, we have

Theorem 1.1. Given $d \geq 2$, let $k$ be the smallest integer such that $k>\max \left(\frac{1}{2}(d+7), d+1\right)$. Assume that $u_{1}^{0}, u_{2}^{0}: \mathbb{R}^{d} \rightarrow \mathbb{R}$ are functions belonging to $H^{\sigma}$ for any $\sigma \geq 0$. Given $q \in(1,2)$ satisfying (4.2), there exists a sufficiently small constant $\epsilon>0$ such that if

$$
\left\|u_{1}^{0}\right\|_{W^{k, 2} \cap W^{2, q}}+\left\|u_{2}^{0}\right\|_{W^{k, 2} \cap W^{2, q}} \leq \epsilon,
$$

then there exists a unique global smooth solution to SMCF of the form (1.3) such that $u_{1}(t)=u_{1}^{0}, u_{2}(t)=u_{2}^{0}$ when $t=0$. Moreover, one has

$$
\langle t\rangle^{\frac{d}{2}\left(\frac{2}{q}-1\right)}\left(\left\|u_{1}(t)\right\|_{W_{x}^{2, \frac{q}{q-1}}}+\left\|u_{2}(t)\right\|_{W_{x}^{2, \frac{q}{q-1}}}\right)+\left\|u_{1}(t)\right\|_{H_{x}^{k}}+\left\|u_{2}(t)\right\|_{H_{x}^{k}} \lesssim \epsilon
$$

for any $t \in \mathbb{R}$. And there exist time independent complex valued functions $\phi_{ \pm} \in H^{2}$ so that

$$
\lim _{t \rightarrow \pm \infty}\left\|u_{1}(t)+\mathrm{i} u_{2}(t)-e^{\mathrm{i} t \Delta} \phi_{ \pm}\right\|_{H_{x}^{2}}=0 .
$$

Theorem 1.1 also shows the $d$-dimensional plane is transversally stable under the SMCF evolution. Let us describe the main idea in the proof of Theorem 1.1. The most challenging problem in dealing with SMCF is that it is highly quasilinear and the leading part is degenerate along the tangent bundle. In the graph like form (1.3), the degenerateness can be avoided using a suitable equivalent formulation of SMFC. The quasilinear nature seems to be unavoidable as far as we know. In fact, whether there exists a gauge transform to make SMCF semilinear is largely open, see Khesin-Yang [16] for some discussions. In this paper, we adopt the strategy dating back to Klainerman [17], the idea is that dispersive estimates of linear part provide time decay of solutions in $L^{p}$ norms with $p>2$ and the high order energy estimates give a chance to overcome the derivative loss in the nonlinear parts. The combination of dispersive estimates and energy estimates can close the bootstrap in the small data case, and thus finishing the proof. The technical part is to choose suitable working spaces in the bootstrap argument.

The local well-posedness also requires some efforts. In fact, [28] raised local wellposedness for non-compact manifold $\Sigma$ as an open problem. The proof of [27] used compactness of second fundamental forms corresponding to a family of immersed manifolds 
with uniform volumes, which is unavailable for general non-compact manifolds. In the $d=2$ and small data case, [27]'s argument indeed works for graph like solutions considered here. But for $d \geq 3$, we need some refinements, because the second fundamental form generally cannot control the graph function $u$ in $d \geq 3$. (See Sec. 3 for more discussions.) In this work, for the local Cauchy problem we can indeed deal with arbitrary large data, see Theorem 3.5 in Section 3. In the view of PDEs, Theorem 3.5 is a local existence and uniqueness theorem for quasilinear Schrödinger equations. There are many works in local well-posedness theory on general quasilinear Schrödinger equations, see for instance the pioneering works by Kenig-Ponce-Vega [12-14], Kenig-Ponce-Rolvung-Vega [11], Marzuola-Metcalfe-Tataru [21-23]. Generally, local well-posedness of quasilinear Schrödinger equations with large data needs additional non-trapping data conditions. Here, due to the geometric structure of SMCF, no non-trapping conditions are needed. This advantage is mainly caused by the high order derivative energy estimates enjoyed by the second fundamental form. In addition, we remark that the well-posedness in the second part of Theorem 3.5 includes the existence of a local solution, uniqueness, and continuous dependence on the initial data.

The paper is organized as follows. In Section 2, we reduce SMCF to a quasilinear Schrödinger equation. In Section 3, we present the local well-posedness and arbitrary order energy estimates. In Section 4, we prove the main theorem for $d \geq 2$. In Section 5 , we finish the whole proof.

Notations. The notation $A \lesssim B$ means there exists some universal constant $C>0$ such that $A \leq C B$. The notation $C_{\gamma_{1}, \ldots, \gamma_{n}}$ denotes some constant depending on the parameters $\gamma_{1}, \ldots, \gamma_{n}$, and generally it varies from line to line. Denote $\langle x\rangle=\sqrt{1+|x|^{2}}$. The Fourier transform $f \mapsto \widehat{f}$ is denoted by

$$
\widehat{f}(\xi)=\int_{\mathbb{R}^{d}} f(x) e^{-i \xi \cdot x} d x .
$$

The usual Sobolev spaces $H^{s}$ are defined by

$$
\|f\|_{H^{s}}=\left\|\langle\xi\rangle^{s} \widehat{f}(\xi)\right\|_{L_{\xi}^{2}}
$$

Let $H^{\infty}=\cap_{s=0}^{\infty} H^{s}$.

\section{Master EQUation}

Let $F: \Sigma \rightarrow \mathbb{R}^{d+2}$, and $\mathbf{H}$ be the mean curvature vector. Then

$$
\Delta_{g} F=\mathbf{H}
$$

where $\Delta_{g}$ denotes the Laplacian on $\Sigma$ of the induced metric $g$ on $T \Sigma_{t}$ given by

$$
g_{i j}=\partial_{x_{i}} F \cdot \partial_{x_{j}} F \text {. }
$$


Since one has

$$
\Delta_{g} F^{\alpha}=g^{i j}\left(\frac{\partial^{2}}{\partial x_{i} \partial x_{j}} F^{\alpha}-\Gamma_{i j}^{l} \frac{\partial}{\partial x_{l}} F^{\alpha}\right),
$$

and the vector $g^{i j} \Gamma_{i j}^{l} \frac{\partial}{\partial x_{l}} F$ belongs to $T \Sigma_{t}$, we see

$$
\mathbf{H}=\left(\Delta_{g} F\right)^{\perp}=\sum_{l=1,2}\left(g^{i j} \frac{\partial^{2} F}{\partial x_{i} \partial x_{j}} \cdot \nu_{l}\right) \nu_{l},
$$

where $\left\{\nu_{l}\right\}_{l=1}^{2}$ denotes the orthonormal basis of $N \Sigma_{t}$ such that $J\left(\nu_{1}\right)=\nu_{2}, J\left(\nu_{2}\right)=-\nu_{1}$. Then, the SMCF can be written as

$$
\partial_{t} F=\left(g^{i j} \frac{\partial^{2} F}{\partial x_{i} \partial x_{j}} \cdot \nu_{1}\right) \nu_{2}-\left(g^{i j} \frac{\partial^{2} F}{\partial x_{i} \partial x_{j}} \cdot \nu_{2}\right) \nu_{1} .
$$

Now, let us consider the case when $F$ is represented by a graph, i.e., $F(x, t)=$ $\left(x_{1}, \ldots, x_{d}, u_{1}, u_{2}\right)$, where $u_{1}, u_{2}$ are functions of $x, t$. It is easy to see the $\left(F^{1}, \ldots, F^{d}\right)$ components of this graph like map $F$ satisfies the afore $d$-equations of SMCF. Set the orthonormal basis $\left\{\nu_{1}, \nu_{2}\right\}$ of $N \Sigma_{t}$ to be

$$
\begin{aligned}
\nu_{1} & =\frac{1}{\sqrt{1+\left|\partial_{x} u_{1}\right|^{2}}}\left(\partial_{x} u_{1},-1,0\right) \\
\nu_{2} & =\frac{1}{\left|\widetilde{\nu}_{2}\right|} \widetilde{\nu}_{2} \\
\widetilde{\nu}_{2} & =\left(\partial_{x} u_{2}, 0,-1\right)-\left[\left(\partial_{x} u_{2}, 0,-1\right) \cdot \nu_{1}\right] \nu_{1},
\end{aligned}
$$

where we denote $\partial_{x} u=\left(\partial_{x_{1}} u, \ldots, \partial_{x_{n}} u\right)$, and $|\cdot|$ denotes the Euclidean norm in $\mathbb{R}^{d}$ or $\mathbb{R}^{d+2}$. Further calculations give

$$
\begin{aligned}
\nu_{2} & =\frac{1}{\Lambda}\left(\partial_{x} u_{2}-\frac{\partial_{x} u_{2} \cdot \partial_{x} u_{1}}{1+\left|\partial_{x} u_{1}\right|^{2}} \partial_{x} u_{1}, \frac{\partial_{x} u_{1} \cdot \partial_{x} u_{2}}{1+\left|\partial_{x} u_{1}\right|^{2}},-1\right) \\
\Lambda & =\left(\left|\partial_{x} u_{2}-\frac{\partial_{x} u_{2} \cdot \partial_{x} u_{1}}{1+\left|\partial_{x} u_{1}\right|^{2}} \partial_{x} u_{1}\right|^{2}+\left|\frac{\partial_{x} u_{1} \cdot \partial_{x} u_{2}}{1+\left|\partial_{x} u_{1}\right|^{2}}\right|^{2}+1\right)^{\frac{1}{2}} .
\end{aligned}
$$

Therefore, the SMCF reduces to

$$
\left\{\begin{array}{l}
\partial_{t} u_{1}=g^{i j}\left(\frac{\partial^{2} F}{\partial x_{i} \partial x_{j}} \cdot \nu_{2}\right) \frac{1}{\sqrt{1+\left|\partial_{x} u_{1}\right|^{2}}}+g^{i j}\left(\frac{\partial^{2} F}{\partial x_{i} \partial x_{j}} \cdot \nu_{1}\right) \frac{\partial_{x} u_{1} \cdot \partial_{x} u_{2}}{\left(1+\left|\partial_{x} u_{1}\right|^{2}\right) \Lambda} \\
\partial_{t} u_{2}=-g^{i j}\left(\frac{\partial^{2} F}{\partial x_{i} \partial x_{j}} \cdot \nu_{1}\right) \frac{1}{\Lambda}
\end{array}\right.
$$

To clarify the main linear part of the above equation, we calculate the expansions of $\nu_{1}, \nu_{2}, \Lambda$. We observe that when $\left|\nabla u_{1}\right|+\left|\nabla u_{2}\right|$ is sufficiently small, $\nu_{1}, \nu_{2}, \Lambda$ have the expansions

$$
\begin{aligned}
\nu_{1} & =\left(\partial_{x} u_{1},-1,0\right)\left[1-\frac{1}{2}\left|\partial_{x} u_{1}\right|^{2}+O\left(\left|\partial_{x} u\right|^{4}\right)\right] \\
\Lambda & =1+O\left(\left|\partial_{x} u\right|^{2}\right) \\
\nu_{2} & =\left(\partial_{x} u_{2}-\frac{\partial_{x} u_{2} \cdot \partial_{x} u_{1}}{1+\left|\partial_{x} u_{1}\right|^{2}} \partial_{x} u_{1}, \frac{\partial_{x} u_{1} \cdot \partial_{x} u_{2}}{1+\left|\partial_{x} u_{1}\right|^{2}},-1\right)\left[1+O\left(\left|\partial_{x} u\right|^{2}\right)\right] .
\end{aligned}
$$


Thus one has

$$
\begin{gathered}
\frac{\partial^{2} F}{\partial x_{i} \partial x_{j}} \cdot \nu_{2}=-\frac{\partial^{2} u_{2}}{\partial x_{i} \partial x_{j}}+O\left(\partial_{x}^{2} u\left|\partial_{x} u\right|^{2}\right) \\
\frac{\partial^{2} F}{\partial x_{i} \partial x_{j}} \cdot \nu_{1}=-\frac{\partial^{2} u_{1}}{\partial x_{i} \partial x_{j}}+O\left(\partial_{x}^{2} u\left|\partial_{x} u\right|^{2}\right) .
\end{gathered}
$$

Now, we see (2.1) reduces to

$$
\left\{\begin{array}{l}
\partial_{t} u_{1}=-g^{i j} \frac{\partial^{2} u_{2}}{\partial x_{i} \partial x_{j}}+O\left(g^{i j} \partial_{i j}^{2} u\left|\partial_{x} u\right|^{2}\right) \\
\partial_{t} u_{2}=g^{i j} \frac{\partial^{2} u_{1}}{\partial x_{i} \partial x_{j}}+O\left(g^{i j} \partial_{i j}^{2} u\left|\partial_{x} u\right|^{2}\right) .
\end{array}\right.
$$

Let us calculate the expansion of $g^{i j}$. In fact, since

$$
g_{i j}=\partial_{x_{i}} F \cdot \partial_{x_{j}} F=\delta_{i j}+\partial_{i} u \cdot \partial_{j} u,
$$

one has by straightforward calculations that

$$
g^{i j}=\delta_{i j}-\frac{\partial_{x_{i}} u \cdot \partial_{j} u}{1+\left|\partial_{x} u\right|^{2}}=\delta_{i j}+O\left(\left|\partial_{x} u\right|^{2}\right) .
$$

Therefore, (2.2) can be written as

$$
\left\{\begin{array}{l}
\partial_{t} u_{1}=-\Delta u_{2}+O\left(\partial_{x}^{2} u\left|\partial_{x} u\right|^{2}\right) \\
\partial_{t} u_{2}=\Delta u_{1}+O\left(\partial_{x}^{2} u\left|\partial_{x} u\right|^{2}\right),
\end{array}\right.
$$

where the $O\left(\partial_{x}^{2} u\left|\partial_{x} u\right|^{2}\right)$ in fact contains many terms including the leading order cubic term like $\partial_{x}^{2} u\left|\partial_{x} u\right|^{2}$ and remainder terms of higher powers of $\left|\partial_{x} u\right|$.

Let $\phi=u_{1}+\mathrm{i} u_{2}$. We see (2.4) is indeed a quasilinear Schrödinger equation with at least cubic interactions:

$$
\mathrm{i} \partial_{t} \phi+\Delta \phi=O\left(\partial_{x}^{2} \phi\left|\partial_{x} \phi\right|^{2}\right)
$$

where the RHS of (2.5) can be written as

$$
\sum c_{i j i^{\prime} j^{\prime}} \partial_{x_{i} x_{j}}^{2} \phi^{ \pm} \partial_{x_{i^{\prime}}} \phi^{ \pm} \partial_{x_{j^{\prime}}} \phi^{ \pm}+\mathcal{R}
$$

Here, $c_{i j i^{\prime} j^{\prime}}$ are universal constants, $\phi^{+}$and $\phi^{-}$denote $\phi$ and $\bar{\phi}$ respectively, and the remainder $\mathcal{R}$ point-wisely satisfies

$$
\left|\partial_{x}^{l} \mathcal{R}\right| \leq C_{l} \sum_{l_{1}+\ldots+l_{j} \leq l, 4 \leq j \leq 2^{10 l}}\left|\partial_{x}^{l_{1}} \partial_{x}^{2} \phi^{ \pm}\right| \ldots\left|\partial_{x}^{l_{j}} \partial_{x} \phi^{ \pm}\right|
$$

for any $l \geq 0$ provided that $\left\|\partial_{x} \phi^{ \pm}\right\|_{L_{t, x}^{\infty}}$ is sufficiently small.

Besides the above approximate equations near 0 , one also has the following exact equation,

$$
\left\{\begin{array}{l}
\partial_{t} u_{1}=-\frac{1}{\Lambda \sqrt{1+\left|\partial_{x} u_{1}\right|^{2}}} g^{i j} \partial_{x_{i} x_{j}}^{2} u_{2} \\
\partial_{t} u_{2}=\frac{1}{\Lambda \sqrt{1+\left|\partial_{x} u_{1}\right|^{2}}} g^{i j} \partial_{x_{i} x_{j}}^{2} u_{1}
\end{array}\right.
$$


which further gives

$$
i \partial_{t} \phi=\frac{1}{\Lambda \sqrt{1+\left|\partial_{x} u_{1}\right|^{2}}} g^{i j} \partial_{x_{i} x_{j}}^{2} \phi
$$

Generally, a further detailed calculation reveals that (2.5)-(2.7) hold as well provided that $\left\|\partial_{x} \phi^{ \pm}\right\|_{L_{t, x}^{\infty}}$ is finite. In fact, we have

Remark 2.1. If $\left\|\partial_{x} \phi^{ \pm}\right\|_{L_{t, x}^{\infty}}<\infty$, then given $l \geq 0$ there exists a constant $C>0$ depending only on $l$ such that (2.5), (2.6) and (2.7) hold.

\section{LOCAL WELL-POSEDNESS AND ENERGy ESTIMATES}

Suppose $F: \mathbb{I} \times \Sigma \rightarrow \mathbb{R}^{d+2}$ is a solution to the SMCF. Recall that for each $t \in \mathbb{I}$, $g=g(t)$ denotes the induced metric on $\Sigma$. Denote the associated volume form on $\Sigma$ by $d \mu=d \mu(t)$. Consider the pullback bundle $F^{*} T \mathbb{R}^{d+2}$ defined over the base manifold $\mathbb{I} \times \Sigma$. This bundle splits naturally into the "spatial" subbundle $\mathfrak{H}$ and the normal bundle $\mathfrak{N}$. Pulling back the metric and connection of $\mathbb{R}^{d+2}$ naturally induces a metric $g^{\mathfrak{N}}$ and connection $\nabla^{\mathfrak{N}}$ defined on the bundle $\mathfrak{N}$, see [1] for a detailed presentation. For simplicity, we write $\nabla$ instead of $\nabla^{\mathfrak{N}}$.

The energy estimates are due to Song-Sun [27] and Song [28]. We recall the results in the following lemma.

Let $F: \mathbb{I} \times \Sigma \rightarrow \mathbb{R}^{d+m}$ be a map given by

$$
F\left(t, x_{1}, \ldots, x_{d}\right)=\left(x_{1}, \ldots, x_{d}, u_{1}\left(t, x_{1}, . ., x_{d}\right), \ldots, u_{m}\left(t, x_{1}, \ldots, x_{d}\right)\right) .
$$

Let $\mathbf{A}$ denote the corresponding second fundamental of $\Sigma_{t}:=\operatorname{Graph}(u)$. Given an integer $l \geq 0$ and a number $p \in[1, \infty)$, define the Sobolev norm of $\mathbf{A}$ by

$$
\|\mathbf{A}\|_{H^{l, p}}=\left(\sum_{k=0}^{l} \int_{\Sigma}\left|\nabla^{l} \mathbf{A}\right|_{g}^{p} d \mu\right)^{\frac{1}{p}} .
$$

If $p=\infty$, we define $\|\mathbf{A}\|_{H^{l, \infty}}$ by

$$
\|\mathbf{A}\|_{H^{l, \infty}}=\sum_{k=0}^{l}\left\|\left|\nabla^{l} \mathbf{A}\right|_{g}\right\|_{L^{\infty}} .
$$

And define the usual Sobolev norm of the Hessian $D^{2} u$ of $u$ by

$$
\left\|D^{2} u\right\|_{W^{l, p}}=\left(\sum_{k=0}^{l} \int_{\Sigma}\left|D^{l} D^{2} u\right|^{p} d x\right)^{\frac{1}{p}} .
$$

Lemma $3.1([27,28])$. With above notions and notations, considering $F: \mathbb{I} \times \mathbb{R}^{d} \rightarrow \mathbb{R}^{d+2}$, there holds point-wisely that

$$
|\mathbf{A}|_{g}^{2} \leq\left|D^{2} u\right|^{2} \leq\left(1+|D u|^{2}\right)^{3}|\mathbf{A}|_{g}^{2}
$$


and for any given $l \geq 0$ there exists a polynomial $Q_{l}$ depending only on $l$ such that

$$
\begin{aligned}
& \left|\nabla^{l} \mathbf{A}\right|_{g} \leq\left|D^{l+2} u\right|+Q_{l}(|D u|) \sum\left|D^{l_{1}+1} u\right| \ldots\left|D^{l_{j}+1} u\right| \\
& \left|D^{l+2} u\right| \leq\left(1+|D u|^{2}\right)^{\frac{l+3}{2}}\left|\nabla^{2} \mathbf{A}\right|_{g}+Q_{l}(|D u|) \sum\left|D^{l_{1}+1} u\right| \ldots\left|D^{l_{j}+1} u\right|,
\end{aligned}
$$

where the summations are taken over the indices $\left(l_{1}, \ldots, l_{j}\right)$ satisfying

$$
l_{1}+\ldots+l_{j}=l+1, l_{1} \geq l_{2} \geq \ldots \geq l_{j}, l \geq l_{i} \geq 1
$$

Let $|D u| \leq \alpha$, then for any $l \geq 0$,

$$
\|\mathbf{A}\|_{H^{l, 2}} \leq C_{\alpha} \sum_{k=1}^{l+1}\left\|D^{2} u\right\|_{W^{l, 2}}^{k}
$$

Let $|D u| \leq \alpha,\left|D^{2} u\right| \leq \beta$, then for any $l \geq 0$,

$$
\left\|D^{2} u\right\|_{W^{l, 2}} \leq C_{\alpha, \beta}\left(\sum_{k=1}^{l}\|\mathbf{A}\|_{H^{l, 2}}^{k}+\sum_{k=2}^{l}\left\|D^{2} u\right\|_{W^{l, 2}}^{k}\right) .
$$

The smooth solution $F(t, x)$ of SMCF satisfies

$$
\frac{d}{d t} \int_{\Sigma}\left|\nabla^{l} \mathbf{A}\right|_{g}^{2} d \mu \leq C \max _{\Sigma}|\mathbf{A}|_{g}^{2} \int_{\Sigma}\left|\nabla^{l} \mathbf{A}\right|_{g}^{2} d \mu
$$

where $C>0$ is a universal constant depending only on $d, l$. Given $p \geq 2$, the smooth solution $F(t, x)$ of SMCF satisfies

$$
\frac{d}{d t} \int_{\Sigma}|\mathbf{A}|_{g}^{p} d \mu \leq C\left(1+\max _{\Sigma}|\mathbf{A}|_{g}^{2}\right) \int_{\Sigma}|\mathbf{A}|_{g}^{p} d \mu+C \int_{\Sigma}|\nabla \mathbf{A}|_{g}^{p} d \mu
$$

where $C>0$ is a universal constant depending only on d,p. Along the SMCF, the induced metric $g$ with $g_{i j}=\partial_{x_{i}} F \cdot \partial_{x_{j}} F$ satisfies

$$
\partial_{t} g_{i j}=-2\left\langle J \mathbf{H}, \mathbf{A}\left(\partial_{x_{i}} F, \partial_{x_{j}} F\right)\right\rangle \text {, }
$$

and the associated volume form $\mu(t)$ satisfies $\partial_{t} \mu(t)=0$.

We remark that although (3.8) was not directly stated in [27, 28], it indeed follows by (3.6). In $d=2,[27]$ proved a stronger result:

$$
\left\|D^{2} u\right\|_{W^{l, 2}} \leq C_{\alpha, \beta} \sum_{k=1}^{l}\|\mathbf{A}\|_{H^{l, 2}}^{k} .
$$

for any $l \geq 0$, if $|D u| \leq \alpha,\left|D^{2} u\right| \leq \beta$. But in $d \geq 3$ one only has (3.8), and (3.12) fails. But (3.8) suffices to work in the small data case by noting that the RHS of (3.8) is at least quadratic in $\left\|D^{2} u\right\|_{W^{l, 2}}$.

We also remark that although $(3.4),(3.7),(3.9)$ were proved in $[27,28]$ for compact $\Sigma$, its proof works for general manifolds where Gagliardo-Nirenberg inequalities hold. 
3.1. Some Sobolev inequalities of tensors. The following two lemmas are useful for graph solutions.

Lemma 3.2. Let $u: \mathbb{R}^{d} \rightarrow \mathbb{R}^{d+2}$ be a graph, $u(x)=\left(x_{1}, \ldots, x_{d}, u^{1}(x), u^{2}(x)\right)$. Suppose that

$$
\|D u\|_{L^{\infty}} \leq \alpha
$$

and the corresponding second fundamental form A satisfies

$$
\|\mathbf{A}\|_{H^{0, \infty}}=\beta<\infty,\|\mathbf{A}\|_{H^{k, 2}}=B_{k}<\infty
$$

for any $k \in \mathbb{Z}_{+}$. Then given $k \in \mathbb{Z}_{+}, p \in[2, \infty)$, there exist an integer $K \in \mathbb{Z}_{+}$depending only on $k, d, p$, and a polynomial $P_{K}$ such that

$$
\left\|D^{2} u\right\|_{W^{k, p}} \lesssim_{\beta, \alpha} P_{K}\left(B_{K}\right) .
$$

Proof. Since $\|D u\|_{L_{x}^{\infty}} \leq \alpha$, the volume $\mu(\cdot)$ induced by immersion $u$ and the usual volume $|\cdot|$ in Euclidean space $\mathbb{R}^{d}$ satisfy

$$
|O| \lesssim_{d, \alpha} \mu(O) \lesssim_{d, \alpha}|O|
$$

for any measurable set $O$ in $\mathbb{R}^{d}$. Given $l \geq 1, p \in[2, \infty)$, integrating (3.6) in $\mathbb{R}^{d}$ we infer from (3.14) that

$$
\left\|D^{l+2} u\right\|_{L^{p}} \lesssim\|\mathbf{A}\|_{H^{l, p}}+C(\alpha) \sum\left\|\left|D^{l_{1}+1} u\right| \ldots \mid D^{l_{j}+1} u\right\|_{L^{p}},
$$

where the summations are taken over the indices $\left(l_{1}, \ldots, l_{j}\right)$ satisfying

$$
l_{1}+\ldots+l_{j}=l+1, l_{1} \geq l_{2} \geq \ldots \geq l_{j}, l \geq l_{i} \geq 1 .
$$

Let's begin with $\left\|D^{2} u\right\|_{L^{2} \cap L^{\infty}}$. By (3.4), one has

$$
\left\|D^{2} u\right\|_{L^{2} \cap L^{\infty}} \lesssim_{\alpha}\|\mathbf{A}\|_{H^{0,2} \cap H^{0, \infty}} \leq_{\alpha} B_{0}+\beta .
$$

The other high derivative terms need more efforts.

Applying Hamilton's interpolation inequality (see Lemma 6.1) to A shows

$$
\int_{\Sigma}\left|\nabla^{j} \mathbf{A}\right|^{\frac{2 i}{j}} d \mu \leq C \max _{\Sigma}|\mathbf{A}|^{2\left(\frac{i}{j}-1\right)} \int_{\Sigma}\left|\nabla^{i} \mathbf{A}\right|^{2} d \mu,
$$

which implies

$$
\|\mathbf{A}\|_{H^{j, \frac{2 i}{j}}} \leq C\left(\alpha, \beta, B_{i}\right)
$$

for any $1 \leq j \leq i$.

Given any $p \in[2, \infty)$, taking $l=1$ in (3.15) we find

$$
\left\|D^{3} u\right\|_{L_{x}^{p}} \lesssim\|\mathbf{A}\|_{H^{3, p}}+C(\alpha)\left\|\left|D^{2} u\left\|D^{2} u \mid\right\|_{L^{p}},\right.\right.
$$

which, together with (3.16) and (3.17), further gives

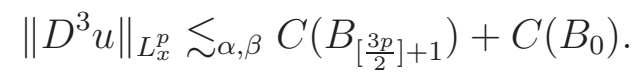


Now, let us prove (3.13) by induction. If for some $k=\nu \geq 1$ (3.13) has been verified, let us prove (3.13) for $k=\nu+1$. For this, taking $l=\nu+1$ in (3.15) we see the summation indexes $\left\{l_{1}, \ldots, l_{j}\right\}$ in the RHS of $(3.15)$ are all smaller than or equal to $\nu+1$. Thus the RHS of (3.15) only involves derivatives of $u$ of order lower than or equal to $\nu+2$. Then by induction assumption, (3.13) also holds in the $k=\nu+1$ case.

Remark 3.3. In the above proof, usual Gagliardo-Nirenberg inequalities cannot be applied to the second fundamental form $\mathbf{A}$, since the embedding constants may depend on the metric $g$ which is also $t$ dependent. So one takes Hamilton's interpolation inequality which is free of metrics.

Lemma 3.4. Let $u: \mathbb{R}^{d} \rightarrow \mathbb{R}^{d+2}$ be a graph, $u(x)=\left(x_{1}, \ldots, x_{d}, u^{1}(x), u^{2}(x)\right)$. Denote $B_{r}(y)$ to be the ball of radius $r>0$ in $\mathbb{R}^{d+2}$ centered at $y \in \mathbb{R}^{d+2}$. Let $\Sigma=u\left(\mathbb{R}^{d}\right)$, and denote the induced metric, measure, the second fundamental form, the mean curvature vector associated with the immersion $u$ by $g, \mu, \mathbf{A}$, and $\mathbf{H}$ respectively. View $\mu$ as a measure in $\mathbb{R}^{d+2}$ with support in $\Sigma$. If $\mu\left(B_{1}(y) \bigcap \Sigma\right)+\|\mathbf{H}\|_{L^{d+\gamma}} \leq D$ for any $y \in \mathbb{R}^{d+2}$ and some $D>0, \gamma>0$, then given $p>d$, for every covariant tensor $\mathbf{T}$ there holds

$$
\max _{\Sigma}|\mathbf{T}|_{g} \leq C\left(\left(\int_{\Sigma}|\nabla \mathbf{T}|_{g}^{p} d \mu\right)^{\frac{1}{p}}+\left(\int_{\Sigma}|\mathbf{T}|_{g}^{p} d \mu\right)^{\frac{1}{p}}\right)
$$

where $C$ depends only on $d, p, \gamma, D$.

Proof. This lemma is essentially due to [Page 157-159, Mantegazza [20]]. We point out that the original result of [20] was stated for compact manifolds $(\mathcal{M}, h)$ with finite volume. But carefully checking [20]'s proof, we see her argument indeed only requires the uniform bounds of $\mu\left(B_{1}(y) \bigcap \Sigma\right)$ for any $y \in \mathbb{R}^{d+2}$ and $\|\mathbf{H}\|_{L^{d+\gamma}}$.

3.2. Local Cauchy problem. Let's move to local well-posedness. Our main result for this section is as follows.

Theorem 3.5. - Given $d \geq 2$, let $k_{0}$ be the smallest integer such that $k_{0}>\frac{d}{2}+2$, i.e. $k_{0}=\left[\frac{d}{2}\right]+3$. Assume that $u_{1}^{0}, u_{2}^{0}: \mathbb{R}^{d} \rightarrow \mathbb{R}$ are functions belonging to $H^{\sigma}$ for any $\sigma \geq 0$. There exists a small constant $T>0$ depending only on

$$
\left\|u_{1}^{0}\right\|_{H^{k_{0}}}+\left\|u_{2}^{0}\right\|_{H^{k_{0}}}
$$

such that there exists a unique smooth local solution F to SMCF of the form

$$
F(t, x)=\left(x_{1}, \ldots, x_{d}, u_{1}(t, x), u_{2}(t, x)\right),\left(u_{1}, u_{2}\right) \uparrow_{t=0}=\left(u_{1}^{0}, u_{2}^{0}\right),
$$

so that $u_{1}, u_{2} \in C\left([-T, T] ; H^{\sigma}\right)$ for any $\sigma \geq 0$.

- Given $d \geq 2$, let $s>\frac{d+5}{2}$. Then there exists $\epsilon>0$ sufficiently small such that for any initial data $u_{0}=\left(u_{1}^{0}, u_{2}^{0}\right)$ with

$$
\left\|u_{0}\right\|_{H^{s}} \leq \epsilon,
$$


the SMCF equation for F of the form (3.20) is locally well-posed in $H^{s}\left(\mathbb{R}^{d}\right)$ on the time interval $[-1,1]$.

We begin with the small data case.

Lemma 3.6. Given $d \geq 2$, let $s>\frac{d+5}{2}$. Then there exists $\epsilon>0$ sufficiently small such that for any initial data $u_{0}=\left(u_{1}^{0}, u_{2}^{0}\right)$ with

$$
\left\|u_{0}\right\|_{H^{s}} \leq \epsilon
$$

the SMCF equation for F of the form (3.20) is locally well-posed in $H^{s}\left(\mathbb{R}^{d}\right)$ on the time interval $[-1,1]$.

Proof. This follows directly by [Theorem 1.1, [21]] and (2.8).

Now, let us prove the local well-posedness theory for arbitrary large data stated in Theorem 3.5. The whole proof is divided into two steps. In Step 1, we prove the existence of solution to SMCF in $t \in[-T, T]$ with $T>0$ depending on

$$
\left\|u_{1}^{0}\right\|_{H^{K}}+\left\|u_{2}^{0}\right\|_{H^{K}}
$$

where $K$ is some integer depending only on $d$ and larger than $k_{0}$. In Step 2, we prove the existence of solution to SMCF in $t \in[-T, T]$ with $T>0$ depending only on

$$
\left\|u_{1}^{0}\right\|_{H^{k_{0}}}+\left\|u_{2}^{0}\right\|_{H^{k_{0}}} .
$$

Lemma 3.7. Given $d \geq 2$, let $k_{0}$ be the smallest integer such that $k_{0}>\frac{d}{2}+2$, i.e. $k_{0}=\left[\frac{d}{2}\right]+3$. Let $\left(u_{1}^{0}, u_{2}^{0}\right) \in H^{\infty}$. Then there exists a constant $T>0$ depending only on

$$
\left\|u_{1}^{0}\right\|_{H^{k_{0}}}+\left\|u_{2}^{0}\right\|_{H^{k_{0}}}
$$

such that there exists a unique smooth local solution F to SMCF of the form

$$
F(t, x)=\left(x_{1}, \ldots, x_{d}, u_{1}(t, x), u_{2}(t, x)\right),\left(u_{1}, u_{2}\right) \uparrow_{t=0}=\left(u_{1}^{0}, u_{2}^{0}\right),
$$

so that $u_{1}, u_{2} \in C\left([-T, T] ; H^{\sigma}\right)$ for any $\sigma>0$.

Proof. Let $\lambda \in(0,1)$. Consider the flow

$$
\partial_{t} F^{\lambda}=J\left(F^{\lambda}\right) \mathbf{H}\left(F^{\lambda}\right)+\lambda \mathbf{H}\left(F^{\lambda}\right), F^{\lambda}(0, x)=\left(x_{1}, \ldots, x_{d}, u_{1}^{0}(x), u_{2}^{0}(x)\right) .
$$

The local well-posedness of (3.21) in $H^{s}$ with $s>\frac{d}{2}+2$ follows by [Prop. 8.1, [29]]. The strong parabolic property of (3.21) can be easily seen from

$$
\left\{\begin{aligned}
\partial_{t} u_{1}^{\lambda} & =\lambda\left(\frac{\left|\partial_{x} u_{1}^{\lambda} \cdot \partial_{x} u_{2}^{\lambda}\right|^{2}}{\Lambda^{2}\left(1+\left|\partial_{x} u_{1}^{\lambda}\right|^{2}\right)^{2}}+\frac{1}{1+\left|\partial_{x} u_{1}^{\lambda}\right|^{2}}\right) g^{i j} \partial_{x_{i} x_{j}}^{2} u_{1}^{\lambda}-\lambda \frac{\partial_{x} u_{1}^{\lambda} \cdot \partial_{x} u_{2}^{\lambda}}{\Lambda^{2}\left(1+\left|\partial_{x} u_{1}^{\lambda}\right|^{2}\right)^{i j}} g_{x_{i} x_{j}}^{2} u_{2}^{\lambda} \\
- & \frac{1}{\Lambda \sqrt{1+\left|\partial_{x} u_{1}^{\lambda}\right|^{2}}} g^{i j} \partial_{x_{i} x_{j}}^{2} u_{2}^{\lambda} \\
\partial_{t} u_{2}^{\lambda} & =\lambda \frac{1}{\Lambda^{2}} g^{i j} \partial_{x_{i} x_{j}}^{2} u_{2}^{\lambda}-\lambda \frac{\partial_{x} u_{1}^{\lambda} \cdot \partial_{x} u_{2}^{\lambda}}{\Lambda^{2}\left(1+\left|\partial_{x} u_{1}^{\lambda}\right|^{2}\right)} g^{i j} \partial_{x_{i} x_{j}}^{2} u_{1}^{\lambda}+\frac{1}{\Lambda \sqrt{1+\left|\partial_{x} u_{1}^{\lambda}\right|^{2}}} g^{i j} \partial_{x_{i} x_{j}}^{2} u_{1}^{\lambda} .
\end{aligned}\right.
$$


We also recall that the solution of $(3.21)$ belongs to $C\left(\left[0, T^{\prime}\right] ; H^{s}\right)$ as long as

$$
\left\|u^{\lambda}\right\|_{L^{\infty}\left(\left[0, T^{\prime}\right] ; C^{\left.1+\delta_{1}\right)}\right.}<\infty \text { for some } \delta_{1}>0 .
$$

Direct calculations show (3.9) also holds for $\mathbf{A}^{\lambda}$, the second fundamental form associated with $F^{\lambda}$. Define $T_{\lambda} \in[0,1]$ to be the maximal time such that

$$
\left\|u^{\lambda}\right\|_{L^{\infty}\left(\left[0, T_{\lambda}\right] ; H^{k_{0}}\right)} \leq C_{0} \varepsilon \ll 1 .
$$

Let $k_{1}=\left[\frac{d}{2}\right]+4$. Define $T_{\lambda} \in[0,1]$ to be the maximal time such that

$$
\left\|u^{\lambda}\right\|_{C\left(\left[0, T_{\lambda}\right] ; H^{k_{1}}\right)} \leq C_{0}\left\|u_{0}\right\|_{H^{K}}
$$

where $K \geq k_{1}$ is an integer depending only on $d$ to be determined later, and $C_{0}>1$ is a constant to be chosen later. By the blow-up criterion and Sobolev embedding, $u^{\lambda}$ is smooth for $t \in\left[0, T_{\lambda}\right]$. We shall use a continuity argument to show $T_{\lambda} \geq T$ for some $T>0$ depending only on $\left\|u_{0}\right\|_{H^{K}}$. shows $u^{\lambda}$ is smooth for $t \in\left[0, T_{\lambda}\right]$.

First, letting $\phi^{\lambda}=u_{1}^{\lambda}+\mathrm{i} u_{2}^{\lambda}$, by Duhamel principle, we infer from (3.24) and the fact $H^{k_{1}-2}$ is an algebra that

$$
\left\|u^{\lambda}\right\|_{C\left(\left[0, T_{\lambda}\right] ; H^{k_{1}-2}\right)} \leq\left\|u_{0}\right\|_{H_{x}^{k_{1}}}+C_{1} T_{\lambda}\left[C_{0}\left\|u_{0}\right\|_{H_{x}^{K}}+C_{0}^{n_{0}}\left\|u_{0}\right\|_{H_{x}^{K}}^{n_{0}}\right],
$$

where $n_{0} \in \mathbb{Z}_{+}$and $C_{1}>0$ depend only on $d$, see Remark 2.1. Second, note that (3.4) with (3.24) implies

$$
\left\|\mathbf{A}^{\lambda}\right\|_{C\left(\left[0, T_{\lambda}\right] ; H^{0, \infty}\right)} \leq C_{2} C_{0}\left\|u_{0}\right\|_{H^{K}}
$$

for some universal constant $C_{2}>0$. Then for any $k \in \mathbb{Z}_{+},(3.9)$, (3.7), and Gronwall inequality give

$$
\left\|\mathbf{A}^{\lambda}\right\|_{C\left(\left[0, T_{\lambda}\right] ; H^{k, 2}\right)} \leq\left(C_{3}\left\|u_{0}\right\|_{H^{k}}+C_{3}\left\|u_{0}\right\|_{H^{k}}^{n_{1}}\right) e^{C_{2} T_{\lambda} C_{0}^{2}\left\|u_{0}\right\|_{H^{K}}^{2}},
$$

where $n_{1} \in \mathbb{Z}_{+}, C_{3}>0$ are independent of $C_{0}$, and depend only on $d, k$. Hence, Lemma 3.2 , (3.27) and (3.24) yield that there exist some sufficiently large $K \in \mathbb{Z}_{+}$depending only on $d$ and a polynomial $P_{K}$ such that

$$
\begin{gathered}
\left\|D^{2} u^{\lambda}\right\|_{C\left(\left[0, T_{\lambda}\right] ; H^{k_{1}-2}\right)} \leq G\left(\left\|D u^{\lambda}\right\|_{C\left(\left[0, T_{\lambda}\right] ; L_{x}^{\infty}\right)}\right) P_{K}\left(\left\|\mathbf{A}^{\lambda}\right\|_{C\left(\left[0, T_{\lambda}\right] ; H^{K, 2}\right)}\right) \\
\leq G\left(\left\|D u^{\lambda}\right\|_{C\left(\left[0, T_{\lambda}\right] ; L_{x}^{\infty}\right)}\right)\left(C_{3}\left\|u_{0}\right\|_{H^{K}}+C_{3}\left\|u_{0}\right\|_{H^{K}}^{n_{2}}\right) e^{C_{2} T_{\lambda} C_{0}^{2}\left\|u_{0}\right\|_{H^{K}}^{2}},
\end{gathered}
$$

where $n_{2} \in \mathbb{Z}_{+}$and $C_{3}>0$ depend only on $k_{0}, d$, and $G: \mathbb{R}^{+} \rightarrow \mathbb{R}^{+}$is of the form

$$
G(y)=C_{4}\left[\left(1+y^{2}\right)^{\frac{k_{1}+3}{2}}+Q_{k_{1}-2}(y)\right]
$$

for some constant $C_{4}>0$ depending only on $d$. Let $n_{3}$ denote the top order of $Q_{k_{0}-2}$. Since $k_{0}>\frac{d}{2}+3,(3.25)$ and Sobolev embedding show

$$
\left\|D u^{\lambda}\right\|_{C\left(\left[0, T_{\lambda}\right] ; L_{x}^{\infty}\right)} \leq C_{5}\left\|u_{0}\right\|_{H^{K}}+C_{5} T_{\lambda} C_{0}^{n_{0}}\left\|u_{0}\right\|_{H^{K}}^{n_{0}}
$$

for some constant $C_{5}>0$ depending only on $d$. Therefore, (3.28) now leads to

$$
\left\|D^{2} u^{\lambda}\right\|_{C\left(\left[0, T_{\lambda}\right] ; H^{k_{1}-2}\right)} \leq e^{C_{2} T_{\lambda} C_{0}^{2}\left\|u_{0}\right\|_{H^{K}}^{2}} C_{6}\left[\left\|u_{0}\right\|_{H^{K}}+\left\|u_{0}\right\|_{H^{K}}^{n_{*}}+\left(T_{\lambda} C_{0}\left\|u_{0}\right\|_{H^{K}}\right)^{n_{*}}\right]
$$


where we denote $n_{*}=n_{0}+n_{1}+n_{2}+n_{3}+k_{1}+3$, and $C_{6}>0$ depends only on $C_{1}, \ldots, C_{5}$ and thus depends only on $k_{0}, d$.

Thus set $C_{0}>1$ to satisfy

$$
C_{0} \geq 100 C_{6}+100\left\|u_{0}\right\|_{H^{K}}^{n_{*}-1}+100
$$

and take $T>0$ to be sufficiently small such that

$$
e^{C_{2} T\left(C_{0}^{2}\left\|u_{0}\right\|_{H}^{2}\right)} \leq 2 ; T C_{0} \leq \frac{1}{2} .
$$

Then by (3.29), (3.25), we get $T_{\lambda} \geq T$ for all $\lambda \in(0,1)$. So there holds

$$
\left\|u^{\lambda}\right\|_{C\left([0, T] ; H^{k_{1}}\right)} \lesssim 1
$$

Therefore, (3.26) shows

$$
\left\|\mathbf{A}^{\lambda}\right\|_{C\left([0, T] ; H^{0, \infty}\right)} \lesssim 1
$$

Hence, (3.9) implies for given $l \geq 0$ there exists $C_{l}>0$ such that

$$
\left\|\mathbf{A}^{\lambda}\right\|_{C\left([0, T] ; H^{l, 2}\right)} \leq C_{l} \text {. }
$$

Step 3. By Lemma 3.2, (3.31), (3.32), $u^{\lambda} \in C\left([0, T] ; H^{k}\right)$ for any given $k \geq 0$ with uniform bounds independent of $\lambda \in(0,1)$. Thus, there exists a sequence $\lambda_{n} \rightarrow 0$ such that $u^{\lambda_{n}}$ converges to $u \in H^{\infty}$ which gives rise to a smooth solution $F$ of SMCF of the form (3.20) in $t \in[0, T]$ with $0<T \ll 1$ fulfilling (3.30). A time reflection and defining $J(F)$ to be the opposite direction rotation can cover $t \in[-T, 0]$ as well.

Step 4. The uniqueness follows by [28]. We remark that although [28] stated uniqueness for compact $\Sigma$, its proof indeed does not use compactness of $\Sigma$. In fact, in [28] the compactness of $\Sigma$ was only used in the existence part.

Remark 3.8. Lemma 3.7 indeed yields an existence theory of strong solutions. In fact, Lemma 3.7 implies there exists an integer $K$ depending only on $d$ such that given an initial data $u_{0} \in H^{K}$, there exists $T>0$ depending only on $\left\|u_{0}\right\|_{H^{K}}$ so that SMCF has a strong solution $u \in L^{\infty}\left([-T, T] ; H^{k_{1}}\right)$ with initial data $u_{0}$. This can be proved by density arguments and the uniform bounds of $\left\|u^{\lambda}\right\|_{L_{t}^{\infty} H_{x}^{k_{1}}}$ obtained in Lemma 3.7. In the case $d=2$, given an integer $l \geq 4$, we can get a strong solution $u \in C\left([-T, T] ; H^{l}\left(\mathbb{R}^{2}\right)\right)$ of SMCF with initial data $u_{0} \in H^{l}\left(\mathbb{R}^{2}\right)$. This improvement is due to the stronger result (3.12) in $d=2$.

To improve Lemma 3.7, we need the following blow-up criterion.

Corollary 3.9. Let $F$ be a local smooth solution to SMCF of the form

$$
F(t, x)=\left(x_{1}, \ldots, x_{d}, u_{1}(t, x), u_{2}(t, x)\right),\left(u_{1}, u_{2}\right) \uparrow_{t=0}=\left(u_{1}^{0}, u_{2}^{0}\right),
$$

such that $u_{1}, u_{2} \in C\left(\left[0, T_{*}\right) ; H^{\infty}\right)$. Then as long as

$$
\|\mathbf{A}\|_{L_{t}^{\infty}\left(\left[0, T_{*}\right) ; H^{0, \infty}\right)}<\infty,
$$


$F$ can be continuously extended to a smooth solution in $\left[0, T_{*}+\rho\right]$ for some $\rho>0$.

Proof. Assume that for some $C_{1}>0$,

$$
\|\mathbf{A}\|_{L_{t}^{\infty}\left(\left[0, T_{*}\right) ; H^{0, \infty}\right)} \leq C_{1}
$$

By (3.11), we get

$$
\partial_{t}|D u|^{2} \leq 2 C_{1}^{2}\left(|D u|^{2}+1\right)
$$

So Gronwall inequality shows

$$
\|D u\|_{L_{t}^{\infty}\left(\left[0, T_{*}\right) ; L_{x}^{\infty}\right)} \lesssim C_{1}, T_{*}\left\|D u_{0}\right\|_{L_{x}^{\infty}}+1
$$

Meanwhile, given $l \in \mathbb{N}$, (3.9) with Gronwall inequality implies there exists $B_{l}>0$ such that

$$
\|\mathbf{A}\|_{L_{t}^{\infty}\left(\left[0, T_{*}\right) ; H^{l, 2}\right)} \lesssim_{T_{*}, C_{1}} B_{l} .
$$

By Lemma 3.2, (3.34) and (3.35) imply for any given $l \in \mathbb{N}$

$$
\left\|D^{2} u\right\|_{L_{t}^{\infty}\left(\left[0, T_{*}\right) ; H_{x}^{l}\right)} \lesssim_{l, T_{*}, C_{1}} 1 .
$$

Integrating (3.33) in $\mathbb{R}^{d}$, we deduce from Gronwall inequality that

$$
\|D u\|_{L_{t}^{\infty}\left(\left[0, T_{*}\right) ; L_{x}^{2}\right)} \lesssim_{C_{1}, T_{*}}\left\|D u_{0}\right\|_{L_{x}^{2}}+1 .
$$

And one has

$$
\frac{d}{d t}\|u\|_{L_{x}^{2}}^{2} \leq\|\mathbf{H}\|_{H^{0,2}}\|u\|_{L_{x}^{2}}
$$

Thus Gronwall inequality yields

$$
\|u\|_{L_{t}^{\infty}\left(\left[0, T_{*}\right) ; L_{x}^{2}\right)} \lesssim C_{1}, T_{*}\left\|u_{0}\right\|_{L_{x}^{2}}+1
$$

In a summary, we get

$$
\|u\|_{L_{t}^{\infty}\left(\left[0, T_{*}\right) ; H_{x}^{k}\right)}<\infty, \forall k \in \mathbb{Z}_{+} .
$$

By Lemma 3.7, $u$ can be continuously extended beyond the interval $0 \leq t<T_{*}$.

Lemma 3.10. Given $d \geq 2$, let $k_{0}$ be the smallest integer such that $k_{0}>\frac{d}{2}+2$, i.e. $k_{0}=\left[\frac{d}{2}\right]+3$. Let $\left(u_{1}^{0}, u_{2}^{0}\right) \in H^{\infty}$. Then there exists a constant $T>0$ depending only on

$$
\left\|u_{1}^{0}\right\|_{H^{k_{0}}}+\left\|u_{2}^{0}\right\|_{H^{k_{0}}}
$$

such that there exists a unique smooth local solution F to SMCF of the form

$$
F(t, x)=\left(x_{1}, \ldots, x_{d}, u_{1}(t, x), u_{2}(t, x)\right),\left(u_{1}, u_{2}\right) \Upsilon_{t=0}=\left(u_{1}^{0}, u_{2}^{0}\right),
$$

so that $u_{1}, u_{2} \in C\left([-T, T] ; H^{\sigma}\right)$ for any $\sigma>0$. 
Proof. It suffices to prove the lifespan in Lemma 3.7 in fact can be improved to depend only on $\left\|u_{0}\right\|_{H^{k_{0}}}$. Given $u_{0} \in H^{\infty}$, by Lemma 3.7 there is a smooth graph solution to SMCF in $t \in\left(-T_{1}, T_{1}\right)$ for some $T_{1}>0$. Let $T_{*}>0$ be the maximal time such that

$$
\|\mathbf{A}\|_{L_{t}^{\infty}\left(\left(-T_{*}, T_{*}\right) ; H^{0, \infty}\right)} \leq C_{*}\left\|u_{0}\right\|_{H^{k_{0}}},
$$

where $C_{*}>1$ is a constant to be determined later. It is easy to see $T_{*}>0$ by the continuity of $u(t)$ in $t$ and Sobolev embeddings. Moreover, we see $T_{*} \leq T_{1}$ by the blow-up criterion in Corollary 3.9. In the rest we prove $T_{*} \geq T$ for some $T>0$ depending only on $\left\|u_{0}\right\|_{H^{k_{0}}}$.

By (3.36), (3.7), (3.9) and Gronwall inequality, we get

$$
\sup _{t \in\left(-T_{*}, T_{*}\right)}\|\mathbf{A}\|_{H^{\left[\frac{d}{2}\right]+1,2}} \leq C_{1} e^{C T_{*} C_{*}^{2}\left\|u_{0}\right\|_{H^{k_{0}}}^{2}}\left(\left\|u_{0}\right\|_{H^{k_{0}}}+\left\|u_{0}\right\|_{H^{k_{0}}}^{m}\right),
$$

where $m \in \mathbb{Z}_{+}, C>0, C_{1}>0$ are universal constants depending only on $d$. At $t=0$, by Sobolev embedding and (3.4), one has

$$
\left\|\mathbf{A}_{0}\right\|_{H^{0,2\left[\frac{d}{2}\right]+2}} \lesssim\left\|u_{0}\right\|_{H^{k_{0}}} .
$$

And applying Hamilton's interpolation inequality (see Lemma 6.1) to A shows

$$
\|\nabla \mathbf{A}\|_{H^{0,2\left[\frac{d}{2}\right]+2}} \leq C_{2}\|\mathbf{A}\|_{H^{0, \infty}}^{1-\frac{1}{\left[\frac{d}{2}\right]+1}}\|\mathbf{A}\|_{H^{\left[\frac{d}{2}\right]+1,2}}^{\frac{1}{\left[\frac{d}{2}\right]+1}}
$$

where $C_{2}>0$ depends only on $d$. Then (3.10) and Gronwall inequality show

$$
\begin{aligned}
& \sup _{t \in\left(-T_{*}, T_{*}\right)}\|\mathbf{A}\|_{H^{0,2\left[\frac{d}{2}\right]+2}}^{2\left[\frac{d}{2}\right]+2} \\
& \lesssim e^{C T_{*}+C T_{*} C_{*}^{2}\left\|u_{0}\right\|_{H^{k_{0}}}^{2}}\left(\left\|u_{0}\right\|_{H^{k_{0}}}^{2\left[\frac{d}{2}\right]+2}+T_{*}\left(C_{*}\left\|u_{0}\right\|_{H^{k_{0}}}\right)^{2\left[\frac{d}{2}\right]}\|\mathbf{A}\|_{L^{\infty}\left(\left(-T_{*}, T_{*}\right) ; H^{\left[\frac{d}{2}\right]+1,2}\right)}^{2}\right),
\end{aligned}
$$

which, together with (3.37), further gives

$$
\sup _{t \in\left(-T_{*}, T_{*}\right)}\|\mathbf{A}\|_{H^{0,2\left[\frac{d}{2}\right]+2}} \leq \Phi\left(T_{*}\left(C_{*}\left\|u_{0}\right\|_{H^{k_{0}}}\right)^{2}+T_{*}\left(C_{*}\left\|u_{0}\right\|_{H^{k_{0}}}\right)^{2\left[\frac{d}{2}\right]},\left\|u_{0}\right\|_{H_{0}^{k}}\right),
$$

for some universal function $\Phi: \mathbb{R}^{+} \times \mathbb{R}^{+} \rightarrow \mathbb{R}^{+}$which is increasing in both variables. For simplicity of notations, we define

$$
\Omega:=T_{*}\left(C_{*}\left\|u_{0}\right\|_{H^{k_{0}}}\right)^{2}+T_{*}\left(C_{*}\left\|u_{0}\right\|_{H^{k_{0}}}\right)^{2\left[\frac{d}{2}\right]} .
$$

Then, (3.40) implies

$$
\|\mathbf{H}\|_{L_{t}^{\infty}\left(\left(-T_{*}, T_{*}\right) ; H^{0,2\left[\frac{d}{2}\right]+2}\right)} \leq \Phi\left(\Omega,\left\|u_{0}\right\|_{H^{k_{0}}}\right) .
$$

Let $B_{1}(y)$ be a unit ball in $\mathbb{R}^{n+2}$. Since $\partial_{t} \mu(t)=0$ along the SMCF, we see for all $t \in\left(-T_{*}, T_{*}\right)$,

$$
\mu_{t}\left(B_{1}(y) \bigcap \Sigma_{t}\right) \leq \mu_{0}\left(\left\{x \in \mathbb{R}^{d}:\left|x-y^{\prime}\right| \leq 1\right\}\right) \leq C\left(\left\|D u_{0}\right\|_{L_{x}^{\infty}}\right) \leq C\left(\left\|u_{0}\right\|_{H_{x}^{k_{0}}}\right),
$$


where $y^{\prime} \in \mathbb{R}^{d}$ is the projection of $y \in \mathbb{R}^{d+2}$ onto $\mathbb{R}^{d}$. By (3.41) and (3.42), we have verified the conditions in Lemma 3.4. So, given $p>d$, Lemma 3.4 implies

$$
\|\mathbf{A}\|_{L^{\infty}\left(\left[-T_{*}, T_{*}\right] ; H^{0, \infty}\right)} \leq C_{1}^{\prime}\left(\|\mathbf{A}\|_{L^{\infty}\left(\left[-T_{*}, T_{*}\right] ; H^{0, p}\right)}+\|\nabla \mathbf{A}\|_{L^{\infty}\left(\left[-T_{*}, T_{*}\right] ; H^{0, p}\right)}\right),
$$

for some $C_{1}^{\prime}>0$ depending only on $p, d$ and $\Omega$. Taking $p=2\left[\frac{d}{2}\right]+2$, we thus obtain by (3.43) and (3.39) that

$$
\|\mathbf{A}\|_{L^{\infty}\left(\left[-T_{*}, T_{*}\right] ; H^{0, \infty}\right)} \lesssim\|\mathbf{A}\|_{L^{\infty}\left(\left[-T_{*}, T_{*}\right] ; H^{0,2\left[\frac{d}{2}\right]+2}\right)}+\|\mathbf{A}\|_{L^{\infty}\left(\left[-T_{*}, T_{*}\right] ; H^{\left[\frac{d}{2}\right]+1,2}\right)},
$$

where the implicit constant depends only on $\Omega$ and $d$. Using Hölder inequality

$$
\|\mathbf{A}\|_{H^{0,2\left[\frac{d}{2}\right]+2}} \leq\|\mathbf{A}\|_{H^{0,2}}^{\frac{1}{\left[\frac{d}{2}\right]+1}}\|\mathbf{A}\|_{H^{0, \infty}}^{1-\frac{1}{\left[\frac{d}{2}\right]+1}}
$$

(3.44) further yields

$$
\|\mathbf{A}\|_{L^{\infty}\left(\left[-T_{*}, T_{*}\right] ; H^{0, \infty}\right)} \lesssim\|\mathbf{A}\|_{L^{\infty}\left(\left[-T_{*}, T_{*}\right] ; H^{\left[\frac{d}{2}\right]+1,2}\right)},
$$

where the implicit constant depends only on $\Omega, d$. Hence, by (3.37) and (3.45) we obtain

$$
\|\mathbf{A}\|_{L^{\infty}\left(\left[-T_{*}, T_{*}\right] ; H^{0, \infty}\right)} \leq \Phi_{1}\left(\Omega,\left\|u_{0}\right\|_{H^{k_{0}}}\right)
$$

for some universal function $\Phi_{1}: \mathbb{R}^{+} \times \mathbb{R}^{+} \rightarrow \mathbb{R}^{+}$which is increasing in both variables.

Now, take $C_{*}$ to be sufficiently large such that

$$
C_{*}\left\|u_{0}\right\|_{H^{k_{0}}} \geq 2 \Phi_{1}\left(1,\left\|u_{0}\right\|_{H^{k_{0}}}\right)+2
$$

and choose $T>0$ to be sufficiently small such that

$$
\Omega=T_{*}\left(C_{*}\left\|u_{0}\right\|_{H^{k_{0}}}\right)^{2}+T_{*}\left(C_{*}\left\|u_{0}\right\|_{H^{k_{0}}}\right)^{2\left[\frac{d}{2}\right]} \leq \frac{1}{2} .
$$

Then (3.46) reveals

$$
\|\mathbf{A}\|_{L_{t}^{\infty}\left([-T, T] ; H^{0, \infty}\right)} \leq \frac{1}{2} C_{*}\left\|u_{0}\right\|_{H^{k_{0}}} .
$$

So one has $T_{*} \geq T$ by (3.36). It is obvious that $T>0$ defined via (3.47)-(3.48) depends only on $\left\|u_{0}\right\|_{H^{k_{0}}}$.

\section{Proof of Theorem 1.1 in $d \geq 2$}

By Duhamel principle, the solution of (2.5) can be expressed by

$$
\phi(t)=e^{\mathrm{i} t \Delta} \phi_{0}+\int_{0}^{t} e^{\mathrm{i}(t-s) \Delta} O\left(\partial_{x}^{2} \phi\left|\partial_{x} \phi\right|^{2}\right)(s) d s .
$$

Let $k$ be the smallest integer such that $k>\max \left(\frac{d}{2}+3.5, d+1\right)$. Let $1<q<2, q^{\prime}=\frac{q}{q-1}$, and

$$
\frac{1}{q}=\frac{1}{d}+\frac{1}{2}-\delta
$$

where $\delta>0$ is sufficiently small. 
Let $\mathcal{T}$ be the maximal time such that

$$
\sup _{t \in[-\mathcal{T}, \mathcal{T}]}\left(\langle t\rangle^{\frac{d}{2}\left(\frac{2}{q}-1\right)}\|\phi(t)\|_{W^{2, q^{\prime}}}+\|\phi(t)\|_{H^{k}}\right) \leq C_{0} \epsilon
$$

where $C_{0}>1$ is to be determined later. Let's prove Theorem 1.1 by bootstrap.

Step 1. By local theorem in Lemma 3.6 and Sobolev embedding, one has $\mathcal{T} \geq 1$.

Step 2. Assume that $1<|t|<\mathcal{T}$, and without loss of generality let $t \in[1, \mathcal{T}]$. Then (4.1) and linear dispersive estimates of $e^{\mathrm{i} \Delta t}$ (see Lemma 6.2) give

$$
\|\phi(t)\|_{W^{2, q^{\prime}}} \lesssim t^{\frac{d}{2}\left(1-\frac{2}{q}\right)}\left\|\phi_{0}\right\|_{W^{2, q}}+\int_{0}^{t} \sum_{l=0}^{2}(t-s)^{\frac{d}{2}\left(1-\frac{2}{q}\right)}\left\|\partial^{l} O\left(\partial_{x}^{2} \phi\left|\partial_{x} \phi\right|^{2}\right)(s)\right\|_{L_{x}^{q}} d s .
$$

1. Leading cubic terms. The leading cubic part of RHS of (4.4) is

$$
\int_{0}^{t}(t-s)^{\frac{d}{2}\left(1-\frac{2}{q}\right)}\left\|\left.\left|\partial_{x}^{3} \phi\right|\left|\partial_{x}^{2} \phi\right| \partial_{x} \phi\left|\left\|_{L_{x}^{q}} d s+\int_{0}^{t}(t-s)^{\frac{d}{2}\left(1-\frac{2}{q}\right)}\right\|\right| \partial_{x}^{4} \phi|| \partial_{x} \phi\right|^{2}\right\|_{L_{x}^{q}} d s
$$

+ low derivative terms.

Gagliardo-Nirenberg inequality shows

$$
\begin{aligned}
& \left\|\partial_{x} \phi\right\|_{L_{x}^{2-q}} \lesssim\|\phi\|_{L_{x}^{q^{\prime}}}^{\omega}\left\|\partial_{x}^{k} \phi\right\|_{L_{x}^{2}}^{1-\omega}, \omega=\frac{k-1-\frac{d}{q^{\prime}}}{k+d\left(\frac{1}{2}-\frac{1}{q}\right)}, \\
& \left\|\partial_{x}^{2} \phi\right\|_{L_{x}^{2-q}} \lesssim\left\|\partial_{x} \phi\right\|_{L_{x}^{q^{\prime}}}^{\theta}\left\|\partial_{x}^{k} \phi\right\|_{L_{x}^{2}}^{1-\theta}, \theta=\frac{k-2-\frac{d}{q^{\prime}}}{k-1+d\left(\frac{1}{2}-\frac{1}{q}\right)} .
\end{aligned}
$$

When $k \geq d+2$, one has for $q$ in (4.2) with $0<\delta \ll 1$,

$$
\begin{aligned}
\frac{d}{2}\left(1-\frac{2}{q}\right) \min (2 \theta, 2 \omega) & <-1 \\
\min (\theta, \omega) & >\frac{1}{2} .
\end{aligned}
$$

Then (4.3) shows (4.5) is dominated by

$$
\begin{aligned}
& \int_{1}^{t}(t-s)^{\frac{d}{2}\left(1-\frac{2}{q}\right)}\left\|\partial_{x}^{4} \phi\right\|_{L_{x}^{2}}\left\|\partial_{x}^{2} \phi\right\|_{L_{x}^{\frac{2 q}{2-q}}}\left\|\partial_{x} \phi \mid\right\|_{L_{x}^{\frac{2 q}{2-q}}} d s+\int_{0}^{1}(t-s)^{\frac{d}{2}\left(1-\frac{2}{q}\right)}\left\|\partial_{x}^{4} \phi\right\|_{L_{x}^{2}}\left\|\partial_{x} \phi\right\|_{L_{x}^{2 q}}^{2-q} d s \\
& \lesssim \epsilon^{3} \int_{1}^{t}(t-s)^{\frac{d}{2}\left(1-\frac{2}{q}\right)} s^{\frac{d}{2}\left(1-\frac{2}{q}\right) 2 \min (\theta, \omega)} d s+\int_{0}^{1}(t-s)^{\frac{d}{2}\left(1-\frac{2}{q}\right)}\|\phi\|_{H^{k}}^{3} d s \\
& \lesssim\langle t\rangle^{\frac{d}{2}\left(1-\frac{2}{q}\right)} \epsilon^{3},
\end{aligned}
$$

provided that $t \in[-\mathcal{T}, \mathcal{T}]$, where we applied Sobolev embedding for $s \in[0,1]$ due to the assumption $k>\frac{d}{2}+3.5$. The low derivatives terms are easier to handle and also contribute to (4.4) by $\langle t\rangle^{\frac{d}{2}\left(1-\frac{2}{q}\right)} \epsilon^{3}$.

2. Remained higher order terms. The higher order remainders are easy to dominate, since we always have by (2.7) that

$$
\int_{0}^{t}(t-s)^{\frac{d}{2}\left(1-\frac{2}{q}\right)}\|\mathcal{R}\|_{W_{x}^{2, q}} d s \lesssim \int_{0}^{t}(t-s)^{\frac{d}{2}\left(1-\frac{2}{q}\right)}\|\phi\|_{W_{x}^{4,2}}\|\phi\|_{W_{x}^{2, \frac{2 q}{2-q}}}^{2}\|\phi\|_{W_{x}^{1, \infty}} d s
$$


which is also admissible by (4.7) and (4.8).

In a summary we have proved in Step 2 that

$$
\|\phi(t)\|_{W^{2, q^{\prime}}} \leq C_{1}\langle t\rangle^{\frac{d}{2}\left(1-\frac{2}{q}\right)}\left[\left\|\phi_{0}\right\|_{W^{2, q}}+C_{0}^{3} \epsilon^{3}\right]
$$

for some $C_{1}>0$ depending only on $d$.

Step 3. Let us deal with the $H^{k}$ norm in (4.3). By (4.9) and Gagliardo-Nirenberg inequality, we see

$$
\left\|D^{2} \phi(t)\right\|_{L_{x}^{\infty}} \lesssim\|\phi\|_{W_{x}^{2, q^{\prime}}}^{\theta_{1}}\|\phi\|_{H_{x}^{k}}^{1-\theta_{1}} \lesssim\langle t\rangle^{\theta_{1} \frac{d}{2}\left(1-\frac{2}{q}\right)} \epsilon
$$

where $\theta_{1} \in(0,1)$ is given by

$$
\theta_{1}=\frac{k-2-\frac{d}{2}}{d\left(\frac{1}{2}-\frac{1}{q}\right)+k-2}
$$

It is easy to verify

$$
\theta_{1} d\left(\frac{2}{q}-1\right)>1
$$

for $k \geq d+2$ and $0<\delta \ll 1$. Hence, for any $t \in[0, \mathcal{T}]$ we get

$$
\int_{0}^{t}\left\|D^{2} \phi(t)\right\|_{L_{x}^{\infty}}^{2} d s \lesssim \epsilon^{2}
$$

Then by (3.4), for $t \in[0, \mathcal{T}]$ we have

$$
\int_{0}^{t} \max _{\Sigma}|\mathbf{A}|_{g}^{2}(s) d s \lesssim \epsilon^{2}
$$

Thus (3.9) and Gronwall inequality show for any $t \in[0, \mathcal{T}]$

$$
\int_{\Sigma}\left|\nabla^{l} \mathbf{A}\right|_{g}^{2}(t) d \mu \leq 2 \int_{\Sigma}\left|\nabla^{l} \mathbf{A}\right|_{g}^{2}(0) d \mu
$$

if $0<\epsilon \ll 1$. Then (3.7) shows for any $t \in[0, \mathcal{T}], 0 \leq l \leq k-2$,

$$
\int_{\Sigma}\left|\nabla^{l} \mathbf{A}\right|_{g}^{2}(t) d \mu \lesssim\left\|\phi_{0}\right\|_{H^{k}} \ll 1
$$

provided that $0<\epsilon \ll 1$. Since the RHS of (3.8) with $l=k-2$ is quadratic in $\left\|D^{2} u\right\|_{W^{k-2,2}}$ and $\left\|D^{2} u\right\|_{W^{k-2,2}}$ is small by bootstrap assumption, (4.11) further implies for any $t \in$ $[0, \mathcal{T}]$

$$
\left\|D^{2} u(t)\right\|_{W^{k-2,2}} \leq C\left\|\phi_{0}\right\|_{H^{k}}
$$

for some $C>0$ if $0<\epsilon \ll 1$. (4.12) provides admissible bounds of $\|\phi\|_{\dot{H}^{l}}$ with $2 \leq l \leq k$. Using (4.1) we get

$$
\|\phi(t)\|_{H_{x}^{2}} \lesssim\left\|\phi_{0}\right\|_{H_{x}^{2}}+\int_{0}^{t} \sum_{l=0}^{2}\left\|\partial^{l} O\left(\partial_{x}^{2} \phi\left|\partial_{x} \phi\right|^{2}\right)(s)\right\|_{L_{x}^{2}} d s .
$$


We observe that the RHS of (4.13) is dominated by $\left\|\phi_{0}\right\|_{H_{x}^{2}}+C_{0}^{3} \epsilon^{3}$ up to a universal constant depending only on $d$ via noting that (4.10) yields

$$
\left\|\partial_{x}^{2} \phi\right\|_{L_{x}^{\infty}}+\left\|\partial_{x} \phi\right\|_{L_{x}^{\infty}} \lesssim \epsilon\langle t\rangle^{-\alpha}
$$

for some $\alpha>\frac{1}{2}$.

As a summary, we have obtained admissible bounds of $\|\phi\|_{H^{k}}$ :

$$
\|u(t)\|_{H^{k}} \leq C_{2}\left[\left\|\phi_{0}\right\|_{H^{k}}+C_{0}^{3} \epsilon^{3}\right]
$$

for some $C_{2}>0$ depending only on $d$ and any $t \in[0, \mathcal{T}]$. The inverse direction $t \in[-\mathcal{T}, 0]$ follows by a time reflection and defining $J(F)$ as the opposite direction rotation. Hence, (4.14) holds for all $t \in[-\mathcal{T}, \mathcal{T}]$.

Step 4. Set $C_{0}$ to satisfy

$$
C_{0}>4+4 C_{1}+4 C_{2}
$$

then choose $\epsilon>0$ to be sufficiently small such that

$$
C_{2} C_{0}^{2} \epsilon^{2} \leq \frac{1}{4}
$$

By Step 1, (4.9) in Step 2, (4.14) in Step 3 and bootstrap, we have proved (4.3) holds with $C_{0} \epsilon$ replaced by $\frac{1}{2} C_{0} \epsilon$. Thus $\mathcal{T}=\infty$. And hence (1.5) holds for all $t \in \mathbb{R}$.

\section{Proof of scattering}

(1.6) is in fact scattering type result. This follows if one has shown

$$
\int_{0}^{\infty}\left\|O\left(\partial_{x}^{2} \phi\left|\partial_{x} \phi\right|^{2}\right)\right\|_{H_{x}^{2}} d s \lesssim 1
$$

But (5.1) has been proved in Step 3 of Section 4.

\section{ApPEndix}

The following is Hamilton's interpolation inequality proved in [[8],Section 12].

Lemma 6.1. Let $T$ be any Tensor defined on manifold $\Sigma$. For $1 \leq j \leq i-1$, there exists a constant $C$ depending only on dimension of $\Sigma$ and $i$, which is independent of the metric $g$ and connection such that

$$
\int_{\Sigma}\left|\nabla^{j} T\right|^{\frac{2 i}{j}} d \mu \leq C \max _{\Sigma}|T|^{2\left(\frac{i}{j}-1\right)} \int_{\Sigma}\left|\nabla^{i} T\right|^{2} d \mu .
$$

The following is linear dispersive estimates.

Lemma 6.2. Let $1 \leq q \leq 2$, and $f \in L^{q}\left(\mathbb{R}^{d}\right)$. Then there exists a constant $C>0$ depending only on $q, d$ such that

$$
\left\|e^{i \Delta t} f\right\|_{L_{x}^{q^{\prime}}} \leq C t^{\frac{d}{2}\left(1-\frac{2}{q}\right)}\|f\|_{L_{x}^{q}}
$$

where $q^{\prime}=\frac{q}{q-1}$. 


\section{ACKNOWLEDGEMENT}

The author owes sincere gratitude to the referees for the insightful comments which have deeply improved the presentation of this work. The author thanks Prof. Chong Song and Youde Wang for drawing the author's attention to SMCF and pointing out an error in the first version of this manuscript. This work is partially supported by NSFChina Grant-1200010237 and Grant-11631007.

\section{REFERENCES}

[1] C. Baker, The mean curvature flow of submanifolds of high codimension, Ph.D. thesis, Australian National University (2010); arXiv:1104.4409.

[2] I. Bejenaru, A. Ionescu, C. Kenig, D. Tataru, Global Schrödinger maps in dimensions d $\geq 2$ : Small data in the critical Sobolev spaces, Ann. Math., 173, 2011, 1443-1506.

[3] N.H. Chang, J. Shatah, and K. Uhlenbeck, Schrödinger maps, Comm. Pure Appl. Math., 53(5), 2000, 590-602.

[4] L. Da Rios, On the motion of an unbounded fluid with a vortex filament of any shape, Rend. Circ. Mat. Palermo 22, 1906, 117-135.

[5] W. Ding, Y. Wang, Local Schrödinger flow into Kähler manifolds, Sci. China Ser. A 44(11), 2001, 1446-1464.

[6] H. Gomez, Binormal motion of curves and surfaces in a manifold, Ph.D. thesis, University of Maryland, 2004.

[7] S. Haller and C. Vizman, Non-linear Grassmannians as coadjoint orbits, Math. Ann. 329(4), 2004, 771-785.

[8] R. Hamilton, Three-manifolds with positive Ricci curvature, J. Differential Geom. 17(2), 1982, 255-306.

[9] H. Hasimoto, A soliton on a vortex filament, J. Fluid Mech. 51, 1972, 477-485.

[10] R. Jerrard, Vortex filament dynamics for Gross-Pitaevsky type equations, Ann. Sc. Norm. Super. Pisa CI. Sci. 1(4), 2002, 733-768.

[11] C.E. Kenig, G. Ponce, C. Rolvung, L. Vega, The general quasilinear ultrahyperbolic Schrödinger equation, Adv. Math., 196(2), 2005, 402-433.

[12] C.E. Kenig, G. Ponce, L. Vega, Small solutions to nonlinear Schrödinger equations, Ann. Inst. H. Poincaré Anal. Non Linéaire, 10, 1993, 255-288.

[13] C.E. Kenig, G. Ponce, L. Vega, Smoothing effects and local existence theory for the generalized nonlinear Schrödinger equations, Invent. Math., 134, 1998, 489-545.

[14] C.E. Kenig, G. Ponce, L. Vega, The Cauchy problem for quasi-linear Schrödinger equations, Invent. Math., 158, 2004, 343-388.

[15] B. Khesin, Symplectic structures and dynamics on vortex membranes, Mosc. Math. J. 12(2), 2012, 46-462.

[16] B. Khesin, C. Yang, Higher-dimensional Euler fluids and Hasimoto transform: counterexamples and generalizations, arXiv preprint, arXiv:1902.08834(2019).

[17] S. Klainerman, Long-time behavior of solutions to nonlinear evolution equations. Arch. Ration. Mech. An., 78(1), 1982, 73-98.

[18] F. Lin, Complex Ginzburg-Landau equations and dynamics of vortices, filaments, and codimension-2 submanifolds, Comm. Pure Appl. Math. 51, 1998, 385-441. 
[19] F. Lin, Topological vorticity and geometric conserved motion, Lecture presented at Workshop on Geometric Partial Differential Equations, Institute for Advanced Study, Princeton, 2009.

[20] C. Mantegazza, Smooth geometric evolutions of hypersurfaces, Geom. Funct. Anal., 12, 2002, 138182.

[21] J.L. Marzuola, J. Metcalfe, D. Tataru, Quasilinear Schrödinger equations II: Small data and cubic nonlinearities, Kyoto J. Math., 54(3), 2012, 179-190.

[22] J.L. Marzuola, J. Metcalfe, D. Tataru D, Quasilinear Schrödinger equations I: Small data and quadratic interactions. Adv. Math., 231(2), 2012, 1151-1172.

[23] J.L. Marzuola, J. Metcalfe, D. Tataru D, Quasilinear Schrödinger equations III: Large Data and Short Time. arxiv preprint, 2020.

[24] J. Marsden and A. Weinstein, Coadjoint orbits, vortices, and Clebsch variables for incompressible fluids, Physica D 7(1-3), 1983, 305-323.

[25] B. N. Shashikanth, Vortex dynamics in $R^{4}$, J. Math. Phys. 53, 2012, 013103.

[26] C. Song, Uniqueness of skew mean curvature flow, Proc. Amer. Math. Soc. 145(11), 2017, 49634970.

[27] C. Song, J. Sun, Skew Mean Curvature Flow. Commun. Contemp. Math. 21(1), 1750090, 2019.

[28] C. Song, Local existence and uniqueness of skew mean curvature flow, arXiv:1904.03822.

[29] M. Taylor, Partial differential equations III, nonlinear equations, 2nd edn., Applied Mathematical Sciences, Vol. 117, Springer, New York, 2011.

[30] C. Terng, Dispersive geometric curve flows, in Surveys in Differential Geometry 2014. Regularity and Evolution of Nonlinear Equations, Surveys in Differential Geometry, Vol. 19, International Press, Somerville, MA, 2015, 179-229.

[31] C. Terng and K. Uhlenbeck, Schrödinger flows on Grassmannians, in Integrable Systems, Geometry, and Topology, AMS/IP Studies in Advanced Mathematics, Vol. 36, American Mathematical Society, Providence, RI, 2006, 235-256.

[32] L. Vega, The dynamics of vortex flaments with corners, http://www.ehu.eus/luisvega/data/ uploads/sapporo-agosto-2014.pdf.

ZE LI

School of Mathematics and Statistics, Ningbo University

Ningbo, 315211, P.R. China

Email address: rikudosennin@163.com 